\title{
Separating Visual and Motor Components of Motor Cortex Activation for Multiple Reach Targets: A Visuomotor Adaptation Study
}

\author{
Tineke Grent-’t-Jong, ${ }^{1,2,3}$ - Robert Oostenveld, ${ }^{2}$ W. Pieter Medendorp, ${ }^{2}$ and Peter Praamstra ${ }^{1,2}$ \\ ${ }^{1}$ Department of Neurology, Radboud University Nijmegen Medical Centre, 6500 HB Nijmegen, The Netherlands, ${ }^{2}$ Donders Institute for Brain, Cognition, \\ and Behaviour, Radboud University Nijmegen, $6500 \mathrm{HE}$ Nijmegen, The Netherlands, and ${ }^{3}$ University of Glasgow, Institute of Neuroscience and Psychology, \\ Glasgow G12 8QB, United Kingdom
}

Ethologically inspired models of movement preparation view the sensorimotor system as sampling information from the environment in a parallel fashion in preparation for multiple potential actions. In support, the configuration of the physical workspace, manipulated by the number or spatial separation of potential targets, has been shown to modulate sensorimotor neural activity. It is unclear, however, whether this modulation is driven by the sensory layout of the workspace or through the associated motor plans. Here, we combine a delayed-movement pre-cuing task with visuomotor adaptation to address this question in human subjects while recording MEG. By dissociating visual and motor coordinates of two targets using visuomotor adaptation, the task was designed to evaluate, in a selective fashion, the effects of visual and movement target separation on movement preparatory activity. The results did not allow the intended comparison due to an unanticipated effect of the direction of visuomotor adaptation on baseline oscillatory power in beta and lowgamma bands. Fortuitously, this effect was dependent on whether the adaptation direction decreased or increased the angular separation between alternative movements. That is, there was a sustained reduction of oscillatory power, which was stronger at small compared with large target separation. These results support a direct influence of movement target separation on motor cortex neural activity, mediated by lateral interactions between simultaneously active motor plans. The results further demonstrate a novel effect of visuomotor adaptation on motor cortex oscillatory activity, with properties that support the local nature of learned changes in visuomotor mapping.

Key words: action selection; magnetoencephalography; motor cortex; neural oscillations; reaching; response preparation

Significance Statement

There is growing evidence that the motor cortex routinely prepares for different movements simultaneously, each suited to a possible course of events in the immediate environment. The preparatory motor cortex activity for different movements can be seen as a competition between groups of neurons. This competition is influenced by how similar the alternative movements are; for example, in terms of direction, determined by the proximity of alternative movement goals. This study investigates whether the proximity of alternative reach goals has a direct influence on motor cortex activity (in the form of brain oscillations) or if it has an effect only through conscious evaluation of the separation between targets. We establish that there is a direct effect, supporting the biased competition model of action selection.

\section{Introduction}

Actions and choices are not masterminded by a central decision faculty in the brain just to be passed on to the motor system for execution. Instead, the sensorimotor system is viewed as sampling information from the environment for a parallel preparation of multiple relevant actions (Cisek and Kalaska, 2010). Key aspects of this view have been elaborated in computational models in which (preuted unpublished reagents/analytic tools; T.G. and P.P. analyzed data; T.G., R.O., W.P.M., and P.P. wrote the paper.

This work was supported by the Netherlands Organization for Scientific Research (Maatschappijen Gedragswetenschappen Grant 404-10-414 to P.P. and W.P.M.). W.P.M. is also supported by the European Research Council (Grant EU-ERC 283567), the Netherlands Organization for Scientific Research (Grant NWO-VICl: 453-11-001), and the European Research Council (EU-FP7-FET Grant SpaceCog 600785). We thank the technical group of the Donders Institute for Brain, Cognition, and Behaviour for the development of the MEG-compatible isometric joystickbased on advice from Richard Carson, Queen's University, Belfast.

The authors declare no competing financial interests.

Correspondence should be addressed to Peter Praamstra, Radboud University Medical Centre, Department of Neurology, P0 Box 9101, 6500 HB Nijmegen, The Netherlands. E-mail: peter.praamstra@radboudumc.nl.

DOI:10.1523/JNEUROSCI.1329-15.2015

Copyright $\odot 2015$ the authors $\quad 0270-6474 / 15 / 3515135-10 \$ 15.00 / 0$ 
)motor cortical activity is pictured as a dynamic neural field consisting of elements coding for a particular movement parameter. Due to cells' individual tuning preferences, alternative movement options shape the field into activation peaks that may partially overlap depending on the distance between the alternative movements in a particular dimension (Erlhagen and Schöner, 2002; Cisek, 2006). The shaping of preparatory activity of cell groups encoding alternative movement choices is dependent on lateral (cooperative and competitive) interactions between neurons.

Evidence for simultaneous preparation of multiple potential actions has been provided by single-unit recordings in monkey (pre)motor cortex (Bastian et al., 2003; Cisek and Kalaska, 2005). Klaes et al. (2011) demonstrated that preparatory activity for multiple actions does not require the actual presence of multiple action goals because an inferred goal is sufficient to elicit it. Pastor-Bernier and Cisek (2011) provided strong evidence for competitive interactions sustaining the representation of multiple actions by showing that the value of an action has no effect on neural activity when only one action is represented, but influences activity relative to the value of a competing action. They also found that competition between actions increased when they were further apart in space, resulting in decreased activity. Evidence from noninvasive human studies is limited. Relevant earlier work found that slow brain potentials (contingent negative variation: $\mathrm{CNV}$ ) and sensorimotor rhythms (theta-, beta-, and low-gamma band power) are sensitive to the manipulation of number and/or spatial separation of alternative movement targets (Praamstra et al., 2009; Tzagarakis et al., 2010; Rawle et al., 2012; Grent-'t-Jong et al., 2013, 2014).

In Grent-'t-Jong et al. (2014), we manipulated spatial proximity of two alternative targets for a joystick pointing movement, revealing stronger delay period sensorimotor cortex preparatory activity in a $30^{\circ}$ compared with a $60^{\circ}$ or $90^{\circ}$ separation condition, evident in increased CNV, beta-ERD, and low-gamma-ERD activity. This pattern conforms to model predictions that at small spatial separation, competition between two movementdirection-encoding groups of neurons is weak due to partial overlap, whereas at large separation, there is strong competition because a choice for one target excludes the alternative (PastorBernier and Cisek, 2011). In the present study, we use dual visuomotor adaptation, separating visual and motor aspects of movement target separation, to address two important issues concerning simultaneous preparation of multiple potential actions.

The issue that motivated the study concerns the relationship between strength of preparatory activity and lateral interactions between competing groups of neurons. Instead of an effect of competition, a modulation in strength of movement preparatory activity may express variation in the inclination or intention to prepare for movement. That is, at a larger separation, subjects will be less inclined to prepare for movement, as supported by behavioral studies on response uncertainty (Bock and Eversheim, 2000; Pellizzer and Hedges, 2003). Visuomotor adaptation was used to distinguish direct effects of spatial target separation (actual spatial layout) on movement preparatory oscillatory activity from effects mediated by the influence that response uncertainty imparts on participants' minds (visually perceived target separation). In addition to this issue, the use of visuomotor adaptation provided the opportunity to address the question of whether simultaneous activation for multiple reach targets reflects parallel activation of motor plans or mere encoding of visual properties of alternative movement targets, as recently addressed in two behavioral studies (Stewart et al., 2014; Gallivan et al., 2015).
The results support a direct influence of movement target separation on motor cortex neural activity that is plausibly mediated by lateral interactions between simultaneously active motor plans. However, they do so in an unanticipated way due to the unexpected effects of visuomotor adaptation on motor cortex oscillatory activity.

\section{Materials and Methods}

Participants. Eighteen healthy, right handed (by self-report) participants (mean age $29.1 \pm 9.1$ years; 6 female) took part in the experiment. Participants were paid 8 Euros per hour for their participation. Written informed consent was provided after explanation of the task and experimental procedures in accordance with local institutional guidelines (Committee on Research Involving Human Subjects, region ArnhemNijmegen, The Netherlands).

Basic experimental setup. The task was a cued center-out movement task with visually presented stimuli and pointing responses made with a custom-built MEG-compatible isometric joystick to one of two pre-cued target locations (cf. Grent-'t-Jong et al., 2014). An experimental trial (Fig. $1 \mathrm{~A})$ started with a pre-cue consisting of one of three possible displays that included a fixation dot in the center of the screen and two alternative movement targets presented at a short distance above it. The two movement target locations were placed symmetrically around the vertical meridian separated from each other by an angle of $30^{\circ}, 60^{\circ}$, or $90^{\circ}$ relative to fixation and movement start point. After $1200 \mathrm{~ms}$, a response cue was presented in the form of a thin white line across the center fixation dot pointing in the direction of either the left or the right target location (Fig. $1 A$, second display). Participants then had to make a joystick pointing movement to the designated location, which had to be completed within the $800 \mathrm{~ms}$ duration of the response-cue presentation. Visual feedback of the joystick movement trajectory was represented by updating the position of a small white cursor dot at every refresh of the screen (running at $60 \mathrm{~Hz}$ ) based on collected real-time $X-Y$ joystick coordinates. Intertrial intervals were, on average, $1750 \mathrm{~ms}$ (randomized between 1500 and 2000 $\mathrm{ms})$, resulting in a mean trial duration of $3750 \mathrm{~ms}$. Stimuli and joystick feedback were presented with Presentation 16.2 software (Neurobehavioral Systems) using a liquid crystal display video projector that was back projected with two front-silvered mirrors onto a translucent screen placed at a distance of $\sim 80 \mathrm{~cm}$ in front of the participant's head.

Experimental conditions. The full MEG experiment was performed as a single session containing four main parts: (1) a dual-adaptation INWARD training, followed by (2) a corresponding INWARD experimental session and (3) a dual-adaptation OUTWARD training, followed by (4) a corresponding OUTWARD experimental session. The order of presentation of INWARD and OUTWARD adaptation subsessions was counterbalanced across participants. INWARD training blocks contained two conditions in which the adapted movement angle between alternative movement goals $\left(30^{\circ}\right.$ and $\left.60^{\circ}\right)$ were smaller than the visual angle $\left(60^{\circ}\right.$ and $90^{\circ}$, respectively) due to adaptation to a $15^{\circ}$ outward rotation of the joystick trajectories [clockwise (CW) for right targets, counterclockwise (CCW) for left targets]. In contrast, in the OUTWARD training blocks, the $15^{\circ}$ rotation of joystick cursor positions was applied inward (i.e., CCW for right and CW for left targets), thus creating an adapted movement angle that was larger (either $60^{\circ}$ or $90^{\circ}$ ) than the presented visual angle between the two alternative movement targets $\left(30^{\circ}\right.$ and $60^{\circ}$, respectively). In the remainder of this article, the two INWARD conditions will be referred to as "VIS60-MOT30" and "VIS90MOT60" and the two OUTWARD conditions as "VIS30-MOT60" and "VIS60-MOT90." This design thus defined four conditions that were combined pairwise. One pair of conditions shared the same visual angle of $60^{\circ}$, but differed in prepared movement angle (either $30^{\circ}$ or $90^{\circ}$ ). The other pair of conditions shared the same prepared movement angle of $60^{\circ}$, but differed in presented visual angle of the two alternative target locations $\left(30^{\circ}\right.$ or $90^{\circ}$ apart $)$.

Paradigm. The dual-adaptation training parts consisted of three phases: (1) a "baseline" phase containing three runs of 32 trials, (2) an "adaptation-training phase" also including three runs of 32 trials, and (3) an "experimental test run" containing one run of 32 experimental trials. 


\section{A GENERAL PARADIGM}

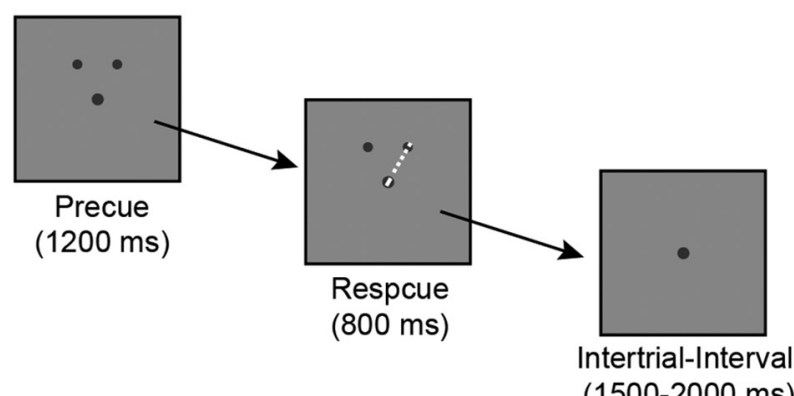

(1500-2000 ms)

\section{B INWARD TRAINING}
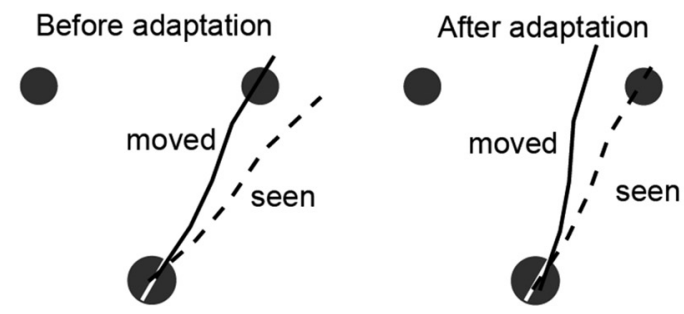

OUTWARD TRAINING

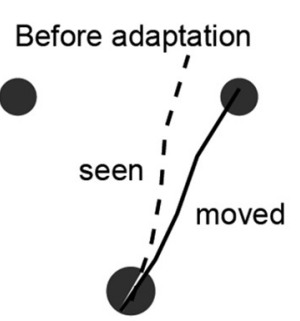

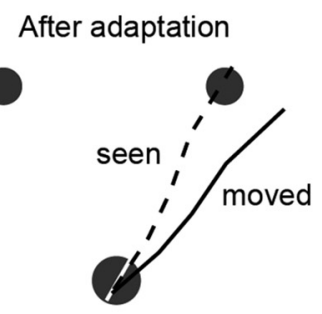

Figure 1. Paradigm. $\boldsymbol{A}$, Timeline of a single trial in the $60^{\circ}$ separation condition. The dashed white lines between the central cue and one of the two peripheral target locations in the response cue display indicates the joystick trajectory. $\boldsymbol{B}$, Example of screen feedback seen by the participants before (left) and after (right) visual-motor adaptation training during the INWARD (top) and OUTWARD (bottom) training blocks. C, Example cursor traces from all correct trials from all four conditions from a representative participant showing trajectories with respect to target locations (black dots) and central starting point.

In all runs, stimuli were randomly drawn from a box-shaped distribution containing equal numbers of rightward and leftward pointing trials from the two included conditions.

During the first phase of the pre-experimental training, the presented computer screens alternated between fixation and response cue displays and participants moved the joystick cursor to the cued location without an intervening delay period. Joystick trajectories in the baseline training phase corresponded to the real joystick movements produced by the participant. During the second phase, real-time joystick $X-Y$ coordinates were rotated by $15^{\circ}$ and presented as cursor position on the screen. Figure $1 B$ shows the effect of this procedure as experienced by the participants during the first few trials in which rotation was applied (Fig. $1 B$, left) and during the end phase after adaptation to the distorted feedback (Fig. $1 B$, right). Finally, in the third phase of the training, a test run was presented in the same format as the subsequently presented experimental blocks; that is, with pre-cue displays added between fixation and response cue displays (as seen in the example trial presented in Fig. $1 \mathrm{~A}$ ). A full training session lasted $\sim 15$ min during the MEG recording session because participants had familiarized themselves with the procedure during an initial $1 \mathrm{~h}$ behavioral training session held on a separate day (1-7 d before the MEG session).

After the INWARD or OUTWARD training, participants received the corresponding experimental block containing 6 runs of 64 trials, each run lasting $\sim 4 \mathrm{~min}$. The first five runs were different randomizations of 64 trials with rotated feedback of the joystick trajectories, resulting in a total of 160 trials per experimental condition; that is, the VIS60-MOT30 and VIS90-MOT60 conditions in the INWARD block and the VIS30MOT60 and VIS60-MOT90 in the OUTWARD block. Data from these five runs with rotation applied were used for the MEG analysis of sensorimotor cortex oscillatory activity. The last run was identical in structure to the first five runs except for a new randomization and no rotation being applied. This last run was used to measure behavioral "deadaptation" effects (angular errors in the opposite direction of the previously applied rotation).

During the entire experiment, participants were asked to move the cursor to the cued location as swiftly as possible after the onset of the response cue while using the highest degree of accuracy without following the cursor trajectories with their eyes. A high degree of accuracy was defined as a leftward or rightward deviation (angular error) from the requested angle of $<10^{\circ}$ (overshooting of the target location was allowed). Movements were always performed with the right hand and had to be made fast and ballistic rather than slow and visually guided. Relaxation of arm and hand was requested during the intertrial and delay periods without releasing the joystick grip or moving the cursor from fixation. Visually presented feedback was provided to the participant after every run, indicating the mean response time and the number of correct trials out of the total number of presented trials in the last run. During the MEG recordings, participants were instructed to move as little as possible and to keep their eyes, which were monitored as well, fixed at the fixation dot at all times (i.e., not following the cursor trajectory).

Recordings. MEG data were recorded continuously using a whole-head system with 275 axial gradiometers (VSM/CTF systems). Head position with respect to the sensor array was measured and monitored during the course of the experiment (Stolk et al., 2013) using localization coils attached to anatomical landmarks (the nasion and, using earplugs, the left and right ear canal). For online monitoring of eye movements, horizontal and vertical electro-oculograms (EOG) were recorded in a bipolar montage using electrodes placed below and above the left eye and at the outer canthi. Joystick position was sampled continuously on the stimulus presentation computer to enable updated joystick cursor positions at screen refresh rate $(60 \mathrm{~Hz})$ during task performance. In addition, the $X-Y$ coordinates were continuously recorded together with the MEG data for accurate response time estimation. All MEG, EOG, and joystick $X-Y$ streaming data were low-pass filtered at $300 \mathrm{~Hz}$, sampled at $1200 \mathrm{~Hz}$, and saved to disk.

Behavioral analysis. Task performance was analyzed on the basis of the joystick position data. Reaction time (RT) for each condition was computed from movement onset, defined as the time when the joystick displacement was first exceeding a threshold of 2.5 SDs above the mean amplitude during a $200 \mathrm{~ms}$ baseline before the response cue. Error analyses were based on an offline trial-by-trial visual inspection of movement trajectories. Errors were classified into three categories: (1) choice and timing errors, (2) corrections, and (3) inaccurately targeted responses. Choice and timing errors included trials in which the end location was at or around the incorrect target, trials in which a response occurred during 

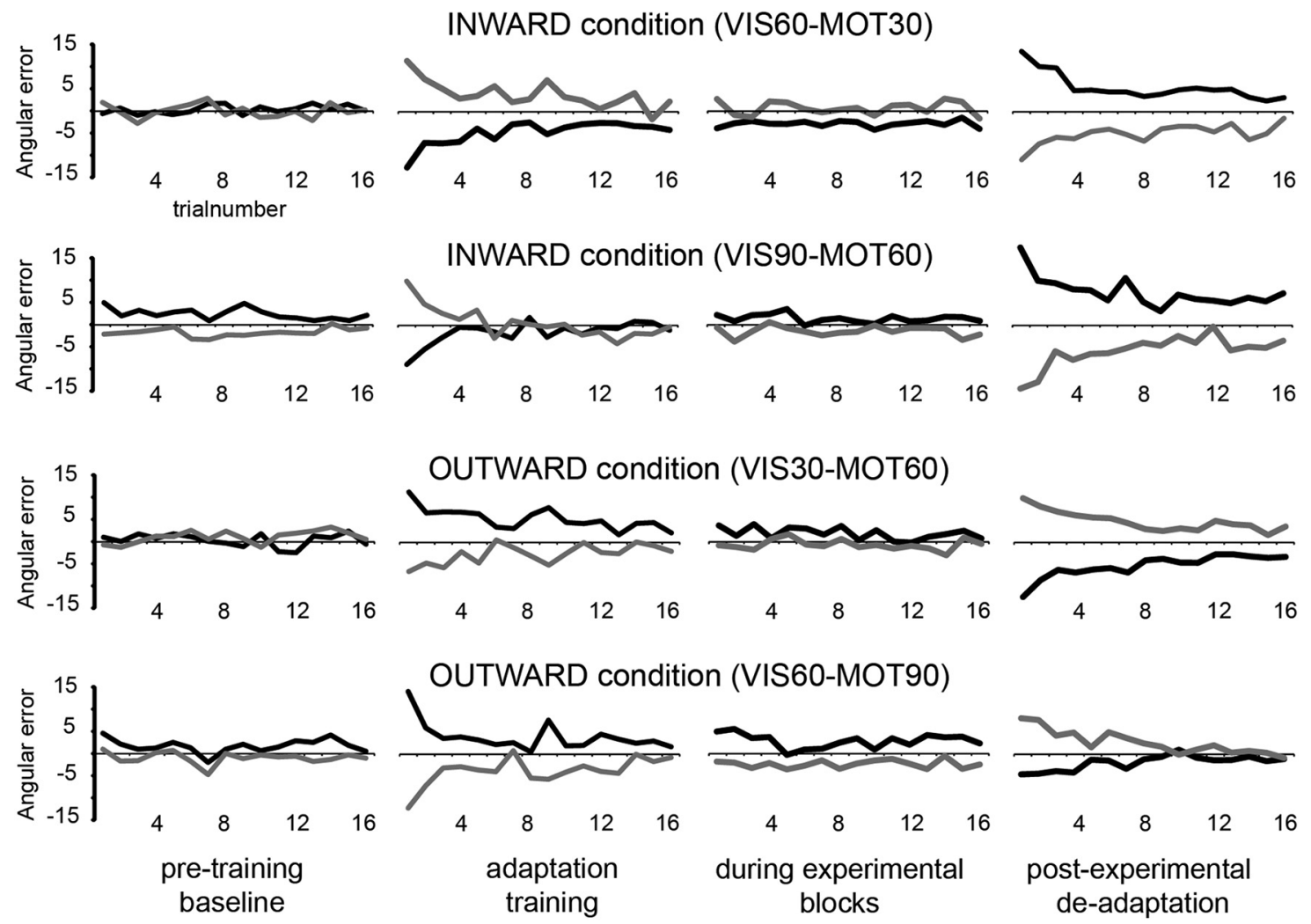
during experimental blocks

\section{post-experimental de-adaptation}

\section{_ RIGHT targets _ LEFT targets}

Figure 2. Behavioral evidence for adequate visual-motor adaptation. Angular errors in four subsequent stages of the experiment. From left to right: column 1 , the last 16 trials from the preadaptation baseline phase; column 2, the first 16 trials from the adaptation training phase; column 3, 16 trials extracted from the second experimental run; and column 4 , the initial 16 trials of the de-adaptation run. Top two rows are from the INWARD condition; bottom two rows from the OUTWARD condition. Black lines, Left-location targets; gray lines, right-location targets. Data were averaged across all participants.

the delay period, and trials in which a response was too fast $(<200 \mathrm{~ms})$, too slow $(>800 \mathrm{~ms})$, or missing altogether. Corrections were trials in which the joystick trajectories revealed online corrections from the incorrect to the correct location, estimated by comparing the cursor position at $50 \%$ and $75 \%$ of computed maximum velocity. Finally, trials of all conditions were classified as "inaccurately targeted" if the target location was missed by $>13^{\circ}$ on either side; that is, a more lenient criterion than used in the online feedback.

Differences in mean RTs between conditions were assessed using repeated-measures ANOVAs, including the within-subject factors ADAPTATION CONDITION (INWARD or OUTWARD) and ANGULAR SEPARATION (narrow or wide). For error rates, repeatedmeasures ANOVAs included the same factors as specified above, but with the additional factor ERRORTYPE (choice and timing error, correction, or inaccurately targeted response). In all analyses, data were averaged across movement direction (left or right).

MEG sensor-data analysis. MEG data were analyzed with MATLAB (The MathWorks) using the open-source Fieldtrip toolbox (Oostenveld et al., 2011). Nonoverlapping epochs of $3900 \mathrm{~ms}$ (1450 ms baseline), centered around pre-cue onset, were extracted separately per condition and for correct response trials only, combining the data from left and right target trials within each condition. Preprocessing included the following steps. First, line noise contamination was removed by applying a digital $50 \mathrm{~Hz}$ discrete Fourier transform filter (including the first two harmonics: 100 and $150 \mathrm{~Hz}$ ) on the continuous data. Second, artifact cleaning was performed, including semiautomatic removal of trials contaminated by muscle activity, slow drift, or SQUID jump artifacts, followed by ICA-based removal of eye-blink, eye movement, and ECG contamination using down-sampled $(300 \mathrm{~Hz})$ data. This resulted in remaining datasets for further analyses consisting of on average 138 trials
$(86.3 \pm 7.6 \%$ rejected) for the VIS60-MOT30, 136 trials $(84.8 \pm 7.5 \%$ rejected) for the VIS90-MOT60, 133 trials ( $83.0 \pm 6.6 \%$ rejected) for the VIS30-MOT60, and 130 trials $(81.3 \pm 7.8 \%$ rejected $)$ for the VIS60MOT90 condition.

Further analyses included time-frequency decompositions performed for horizontal and vertical planar transformed MEG data that was subsequently combined to obtain the power at each virtual planar gradiometer location regardless of the orientation of the gradient (Bastiaansen and Knösche, 2000). This procedure simplifies the interpretation of the sensor-level data because, with planar gradients, the maximal signal is located above the source (Hämäläinen et al., 1993). Time-frequency power representations (TFRs) were computed based on a sliding window Fourier approach with a step size of $50 \mathrm{~ms}$. Power of lower frequencies (range 1-60 Hz) were estimated based on $4 \mathrm{~s}$ of padded data (original length $3.9 \mathrm{~s}$ ) using an adaptive sliding window of 3 cycles per frequency bin (step-size $1 \mathrm{~Hz}$ ), multiplying the data with a Hanning taper before power estimation. From these data, beta $(17-29 \mathrm{~Hz})$ power estimates were extracted for further analysis. For the higher-frequency range, a multitapering approach was used with orthogonal Slepian tapers (Mitra and Pesaran, 1999), which allows better capture of the broadband oscillatory activity in the higher range while reducing spectral leakage. For this frequency range $(30-120 \mathrm{~Hz}$; step-size $5 \mathrm{~Hz})$, power was estimated using sliding windows of 30 cycles per frequency bin and applying a spectral smoothing of $10 \mathrm{~Hz}$ by adapting the number of orthogonal Slepian tapers (2-9 tapers; higher number used for lower frequencies and vice versa). From these data, low-gamma-band $(30-50 \mathrm{~Hz})$ power estimates were extracted for further analysis.

$M E G$ statistical analysis. The two main contrasts that were tested represented the effect of perceived visual angle between the two alternative movement target locations (VIS-effect: VIS30-MOT60 - VIS90- 

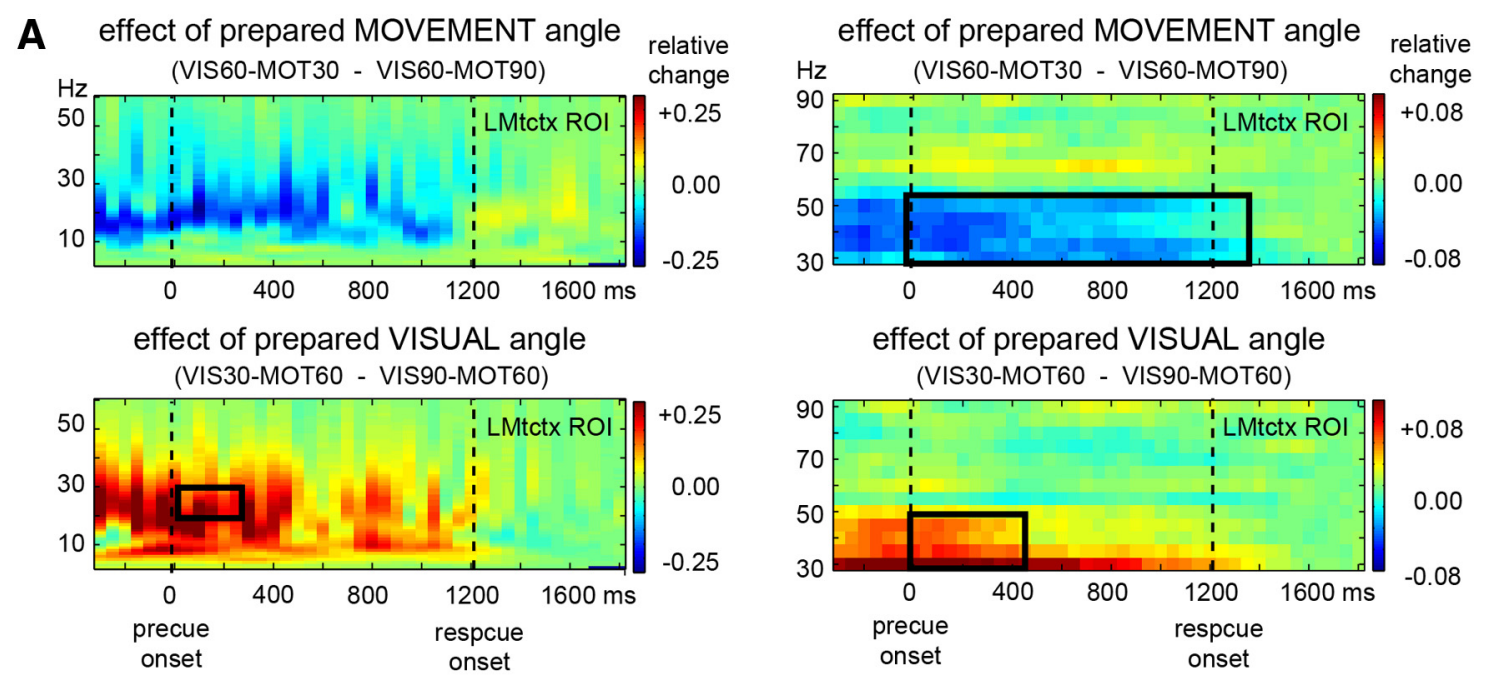

effect of prepared VISUAL angle

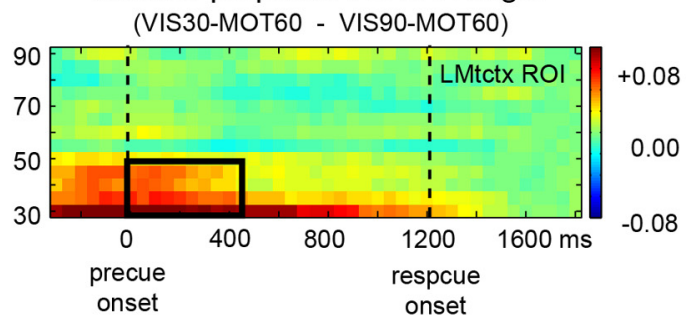

B
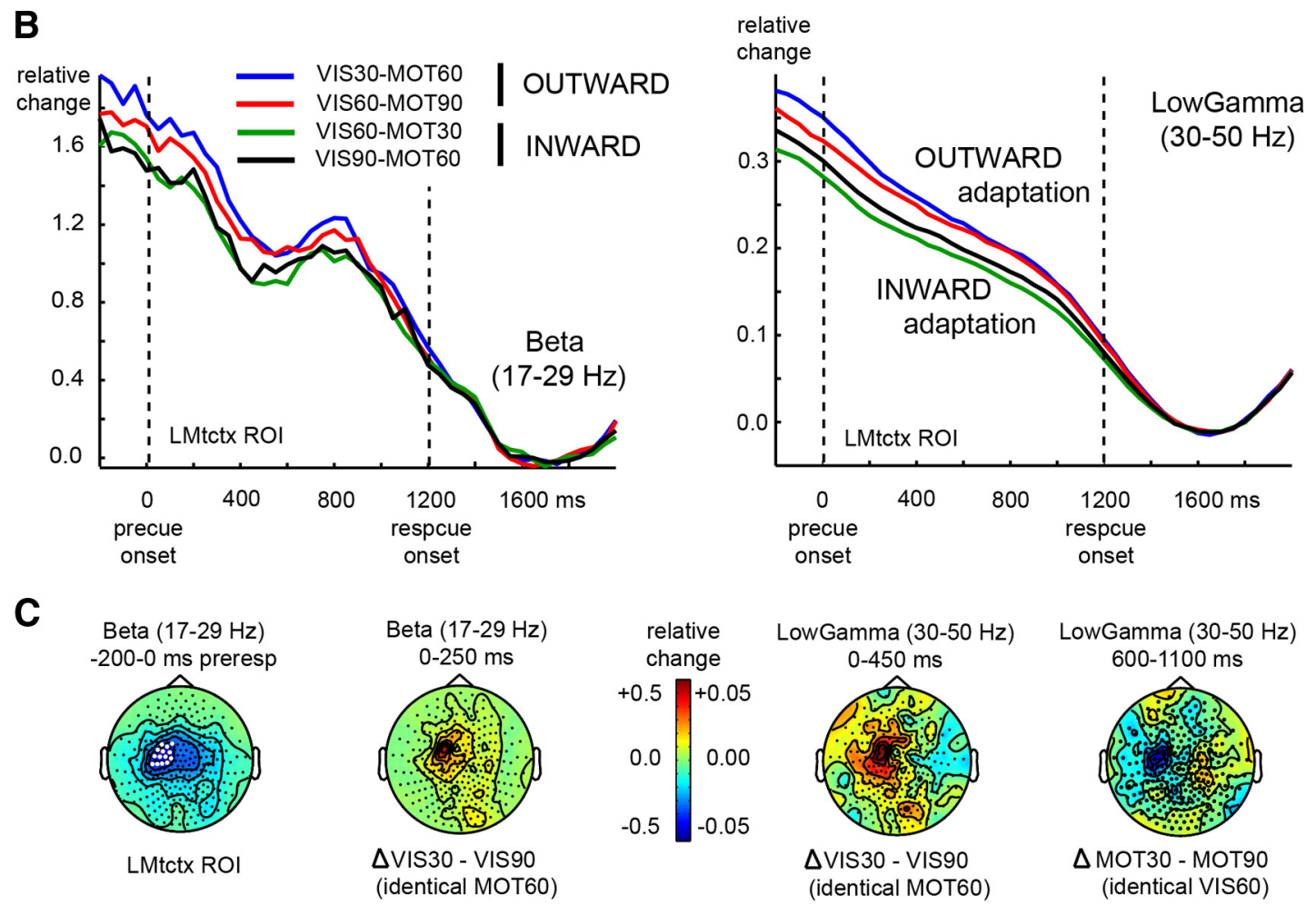

Figure 3. MEG activity. A, Grand-average TFRs of lower-frequency range (1-60 Hz: left two figures) and higher-frequency range ( $30-90 \mathrm{~Hz}$ : right two figures) of data averaged across left sensorimotor cortex sensors (LMtctx ROI, C, left-most figure). Top row TFRs represent the effect of movement angle, lower row TFRs show effect of visual angle. Black boxes indicate windows of significant changes in power from baseline. B, LMtctx ROI-derived grand-average traces of relative changes in beta (left) and low-gamma band (right) power separately per condition. Note the divergence between traces of the INWARD and OUTWARD adaptation conditions, indicating an overlapping effect due to the direction of adaptation. $C$, Left to right, Grand-average topographical distributions of preresponse $(-200-0 \mathrm{~ms}$ ) beta-ERD activity (with white dots representing the LMtctx ROI sensors); the early transient beta-band effect of visual angle ( $0-250 \mathrm{~ms})$; the early transient low-gamma band effect of visual angle $(0-400 \mathrm{~ms})$; and the sustained delay-period low-gamma band effect of movement angle $(600-1100 \mathrm{~ms}$, representative of $0-1300 \mathrm{~ms}$ window of significance). All distributions show maximum effects over the sensorimotor cortex contralateral to the hand used for joystick control.

MOT60 condition) and the effect of prepared movement angle for pointing to one of the cued target locations (MOT-effect: VIS60MOT30 - VIS60-MOT90 condition). Task-related changes in power in the frequency bands of interest were computed as relative changes from baseline by subtracting the mean baseline power from single time point power values across time and dividing them by the same mean baseline power. The resulting values then represent baseline-normalized power changes, with 1 representing a 100\% increase and -1 a 100\% decrease in power from baseline. Unexpectedly, differences in power between conditions in the frequency bands of interest were already present in the intertrial interval. We therefore used a baseline defined around the peak desynchronization coinciding with the response onset (1600-1900 ms after the pre-cue onset). Although this choice of baseline is uncommon, it was also used in related work by Tzagarakis et al. (2010). The choice is justified by the general finding that peak desynchronization levels accompanying a motor response are generally identical across conditions, even when experimental manipulations induce pronounced power differences during the preceding delay period (Doyle et al., 2005; van Wijk et al., 2009; Pastötter et al., 2012).

Significant effects of the visual or the movement angle across time were assessed using cluster-based nonparametric permutation statistics (Maris and Oostenveld, 2007) on beta- (17-29 Hz) and low-gamma- 

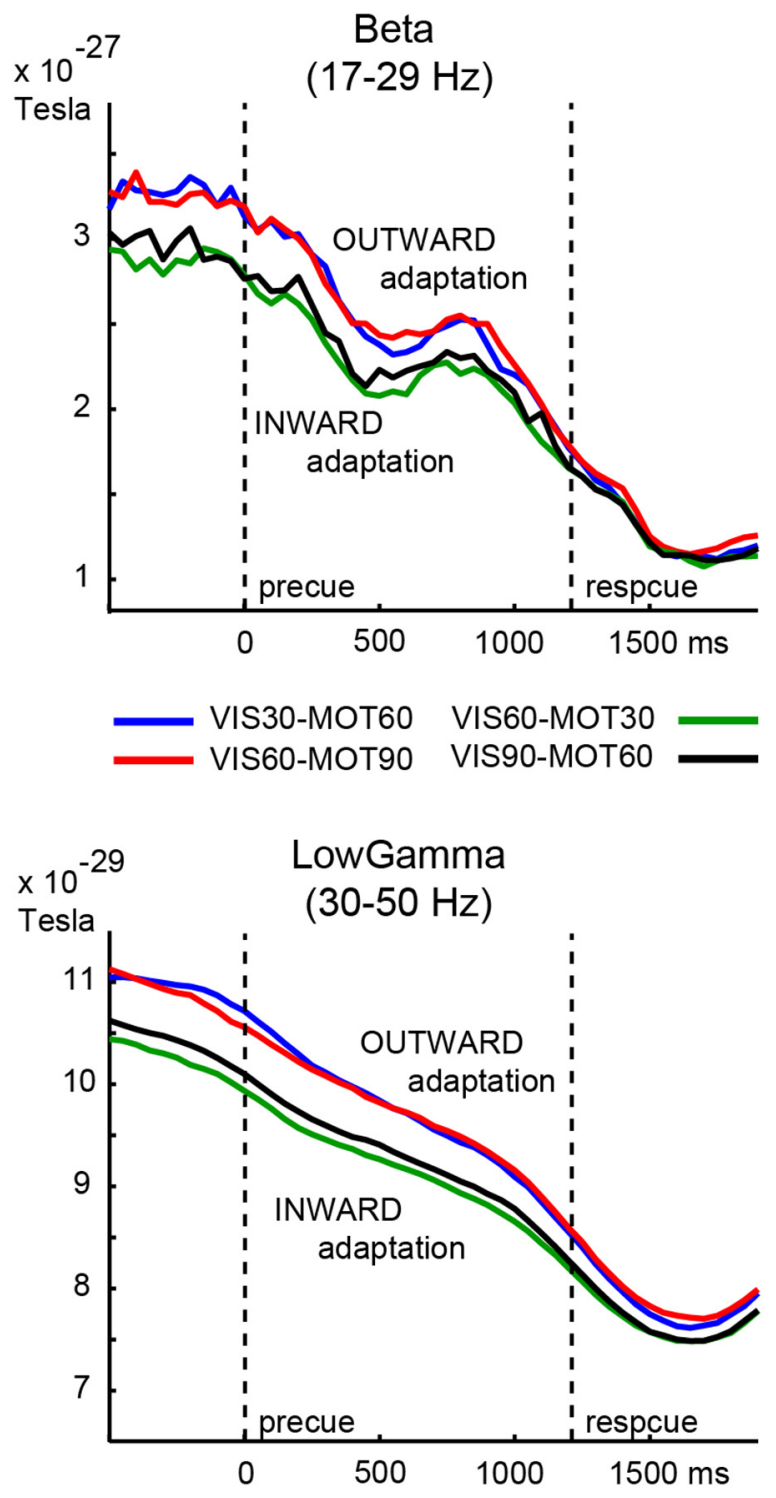

Figure 4. Grand-average traces of relative changes in beta (top) and low-gamma band (bottom) power of the LMtctx ROI. Same data as in Figure 3B, but before application of a common baseline, to show segregation of inward and outward adaptation conditions.

band $(30-50 \mathrm{~Hz})$ power values averaged across a small group of sensors covering the left sensorimotor-cortex. This left sensorimotor cortex region-of-interest (LMtctx ROI) contained a selection of 14 sensors with maximum grand average $(n=18)$ preresponse $(-200$ to $0 \mathrm{~ms}$; response locked) beta power suppression (see Fig. $3 C$, far left) measured against a $500 \mathrm{~ms}$ baseline (1000-500 ms before response onset). The ROI fully overlapped with the LMtctx ROI previously reported for a different group of participants in a comparable study (Grent-'t-Jong et al., 2014). Time points included in the analyses covered the entire delay and most of the response window $(0-1800 \mathrm{~ms}$, pre-cue time locked).

The cluster-based nonparametric permutation test is well suited for controlling the false-alarm rate when facing multiple comparisons, in this case caused by contrasting two conditions for multiple time points. The procedure includes the following steps. First, two distributions are created by random partitioning of trials drawn from the combined dataset, including trials from both experimental conditions. Second, test statistics is calculated on this random partition. These two steps are then repeated a number of times (here, 1000 times) to construct a histogram of the test results. From the actually observed test results and the created histogram, the proportion of random partitions is then calculated that shows larger test statistics than the observed one. This proportion is expressed as $p$ value. Significant differences between the original experimental conditions in this study were assumed when the computed $p$ value was smaller than the critical $\alpha$-level of 0.05 . The threshold to include a time-frequency element into a cluster was $p<0.05$. A minimum cluster size of two adjacent time points (spanning $100 \mathrm{~ms}$ ) was required before a difference between conditions was accepted as significant.

\section{Results}

\section{Behavioral performance}

The task was performed with a high degree of accuracy. The number of trials rejected due to inaccuracy in pointing direction (category 3 of possible error types) was on average 5 trials $(2.8 \pm$ $3.4 \%$ rejected) for the VIS60-MOT30, 6 trials $(3.9 \pm 3.4 \%$ rejected) for the VIS90-MOT60, 8 trials $(4.8 \pm 2.5 \%$ rejected) for the VIS30-MOT60, and 13 trials $(8.2 \pm 4.6 \%$ rejected $)$ for the VIS60-MOT90 condition out of 160 trials per condition. The total number of rejected trials, including also the other two error categories (choice and timing errors and corrected trials) was on average 7 trials $(4.1 \pm 3.8 \%$ rejected $)$ for the VIS60-MOT30, 8 trials $(5.1 \pm 3.5 \%$ rejected $)$ for the VIS90-MOT60, 12 trials $(7.5 \pm 4.3 \%$ rejected) for the VIS30-MOT60, and 15 trials $(9.1 \pm$ $5.0 \%$ rejected) for the VIS60-MOT90 condition. Therefore, very small numbers of trials were rejected for reasons other than directional inaccuracy.

Participants were in general less accurate in the OUTWARD compared with the INWARD experimental conditions $\left(F_{(1,17)}=\right.$ $19.0, p<0.001)$. This decrease in accuracy was primarily caused by an increase in accuracy errors rather than of the other two error types, corresponding with a main effect for ERRORTYPE $\left(F_{(2,34)}=48.3, p<0.001\right)$ and a two-way interaction effect for ADAPTATION CONDITION $\times$ ERRORTYPE $\left(F_{(2,34)}=9.4\right.$, $p<0.001)$. An interaction for ANGULAR SEPARATION $\times$ ERRORTYPE $\left(F_{(2,34)}=16.2, p<0.001\right)$ was explained by participants producing more inaccurately pointed responses with increasing angular separation.

Response times were comparable across conditions: $563 \pm 34$ $\mathrm{ms}$ (SD of the mean) for the VIS60-MOT30, $567 \pm 31 \mathrm{~ms}$ for the VIS90-MOT60, $559 \pm 32 \mathrm{~ms}$ for the VIS30-MOT60, and $565 \pm$ $29 \mathrm{~ms}$ for the VIS60-MOT90 condition. Despite the small difference of at most $8 \mathrm{~ms}$ between conditions, repeated-measures ANOVAs revealed a main effect for ANGULAR SEPARATION $\left(F_{(1,17)}=12.1, p=0.003\right)$. In general, in both the INWARD and OUTWARD experimental blocks, participants responded more slowly when alternative target locations were wider apart compared with when the angle between them was smaller.

Crucial for the interpretation of the neurophysiological results is whether participants adequately adapted to the applied rotation of the visual joystick position feedback signal. One source of evidence is presented in Figure $1 C$-all cursor trajectories are straight and in the cued direction-but the main evidence is presented in Figure 2. In this figure, differences (angular errors) between observed movement angles (estimated at peak velocity) and requested angles for rightward and leftward pointing movements are displayed for 16 trials (averaged across all participants) extracted from four subsequent stages within the full experimental design, namely: (1) the last 16 trials from the preadaptation baseline phase (Fig. 2, first column from left), (2) the first 16 trials from the adaptation training phase (Fig. 2, second column), (3) 16 trials extracted from the second experimental run (Fig. 2, third column), and (4) the initial 16 trials after the start of the de-adaptation run (Fig. 2, rightmost column). As the figure demonstrates, angular errors were very comparable across baseline (preadaptation) runs and experimental runs (fully 


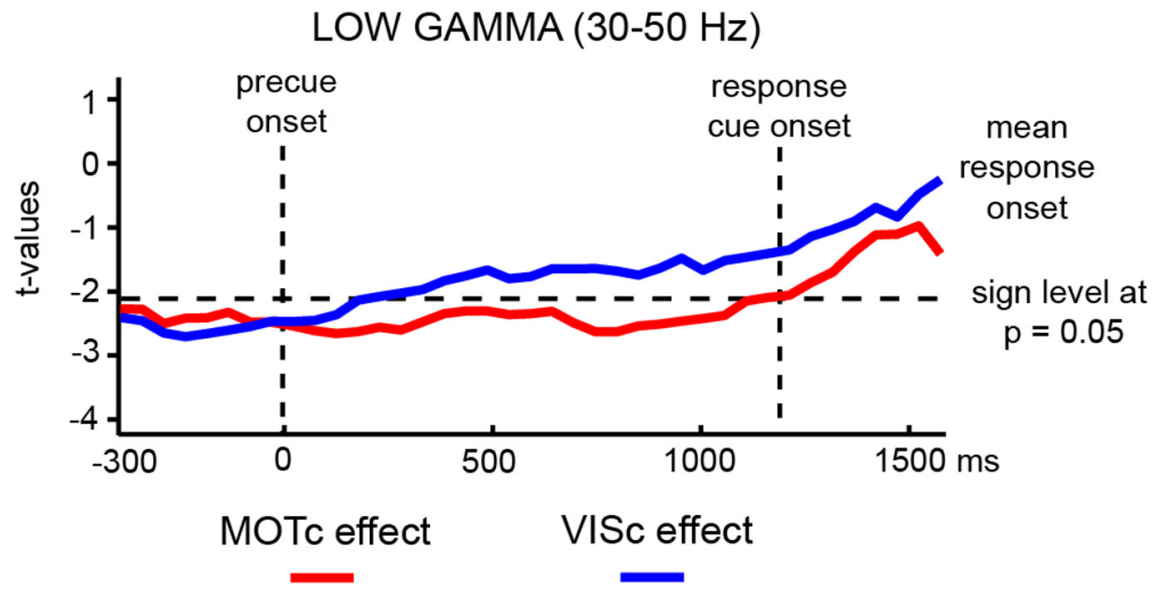

Figure 5. Time course of the VISc and MOTc effects in terms of $t$ values determined in 50 ms windows (VIS and MOT effects recognized to be confounded by an effect of the direction of visuomotor adaptation). After the pre-cue, the VISc effect tapers off faster than the MOTc effect.

adapted), but deviated for the first few trials, with opposite polarity for rightward and leftward trials, during adaptation and de-adaptation phases. This is the expected pattern for adaptation to a visual-motor rotation (Hinder et al., 2010, Woolley et al., 2011).

\section{Perceived angle and movement angle effects on sensorimotor oscillatory activity}

Our main question concerned the nature of the effect of target separation on movement preparatory oscillatory activity. We investigated to what extent this known effect is a direct reflection of physical changes in the workspace or dependent on the visual appreciation of the target distance. To isolate the effect of prepared movement angle (MOT-effect), the two conditions with identical visual angle were subtracted (VIS60MOT30 - VIS60-MOT90). Likewise, to evaluate the effect of visually perceived target separation (VIS-effect), the two conditions with identical movement angle were subtracted (VIS30-MOT60 - VIS90-MOT60).

As explained in the Materials and Methods, the presence of a sustained difference in oscillatory power between conditions necessitated a choice of baseline around the peak beta and lowgamma desynchronization, coinciding with the response onset (1600-1900 ms after the pre-cue onset). Using this alignment of conditions, computation of the VIS effect showed a significant effect in the window $0-250 \mathrm{~ms}$ for beta power $(p<0.05)$ and in the window $0-450 \mathrm{~ms}$ for low-gamma power $(p<0.05)$. The MOT effect was significant in the window $0-1350 \mathrm{~ms}$ for lowgamma power $(p<0.05)$ (Fig. $3 A)$. These effects were localized to the motor cortex, as shown in Figure $3 C$.

Note that the VIS and MOT effects of relative power change during the delay period were of opposite sign, as illustrated in the time-frequency spectrograms of Figure $3 A$. The background of this remarkable polarity change is illustrated in Figure $3 B$ with line plots of the relative power changes in beta and low-gamma bands. For the two conditions that form the basis of the MOT effect, the one with the smallest angle (VIS60-MOT30) shows lower power during the intertrial interval and delay period than the condition with the larger angle (VIS60-MOT90), both for the beta-band and the low-gamma band. For the VIS effect, the amplitude relation is reversed, with the condition with largest angle (VIS90-MOT60) showing lower power than the condition with the small angle (VIS30-MOT60).
The opposite sign of MOT and VIS effects indicates opposite effects on movement preparatory beta and low-gamma activity, which is an implausible result. Exploring possible explanations, we inspected the beta and low-gamma time courses before application of a common baseline. As shown in Figure 4, this representation reveals that the four conditions segregate in two groups according to the direction of adaptation, inward or outward. The magnitude of this effect of the direction of adaptation is substantial and overlaps with the VIS and MOT effects. The overlap would not prohibit comparison of VIS and MOT effects if it was not the case that VIS and MOT effects map onto OUTWARD-INWARD and INWARD-OUTWARD direction of adaptation, respectively. This explains the opposite sign of VIS and MOT effects and implies that the confound prohibits selective evaluation of VIS and MOT effects.

This account of VIS and MOT effects being confounded by an overlapping effect of visuomotor adaptation might be evident in a divergence in time course of the confounded VIS and MOT effects (labeled VISc and MOTc). That is, with a stronger taskrelated power suppression at small compared with large (visual and/or movement) angle, the (absolute) size of the VISc effect should reduce faster than the size of the MOTc effect from the time of presentation of the pre-cue. Figure 5 shows that this is indeed the case for low-gamma power (Fig. 5). It was not evident in the task-related beta modulation. Both results are in agreement with the timing of VIS and MOT effects summarized in Figure $3 A$.

\section{Nature of the effect of visuomotor adaptation during the intertrial period}

The unusual finding of differences in power between conditions already during the intertrial period seem related to visuomotor adaptation, but are not yet explained. As shown, the four conditions segregate in two groups according to the direction of adaptation. This segregation, implying a sustained difference between conditions, may be due to persistent motor cortical activity associated with visuomotor adaptation, as described by Paz et al. (2003), in the form of increased activity of cell populations encoding the adapted (learned) movement direction. We hypothesize that this increased activity, in conjunction with the here investigated oscillatory effects of target separation, causes the difference in power between INWARD and OUTWARD adapted conditions during the intertrial period. That is, both inward and outward adaptation induce increased activity of cell populations encoding the learned movements, but the learned movement directions have a smaller angular separation in the INWARD conditions compared with the OUTWARD conditions. Therefore, there is stronger persistent activity in the former conditions, with a resulting lower power in beta and gamma bands.

Complementary analyses of the data acquired during the adaptation training afforded an opportunity to test this hypothesis, albeit limited by a small number of trials and absence of the delay period. We compared the data acquired during training phase 1 [last 64 of $96(3 \times 32)$ trials without adaptation] with data acquired during training phase 2 [last 64 of $96(3 \times 32)$ trials with adaptation]. Our hypothesis entails that the baseline power be- 

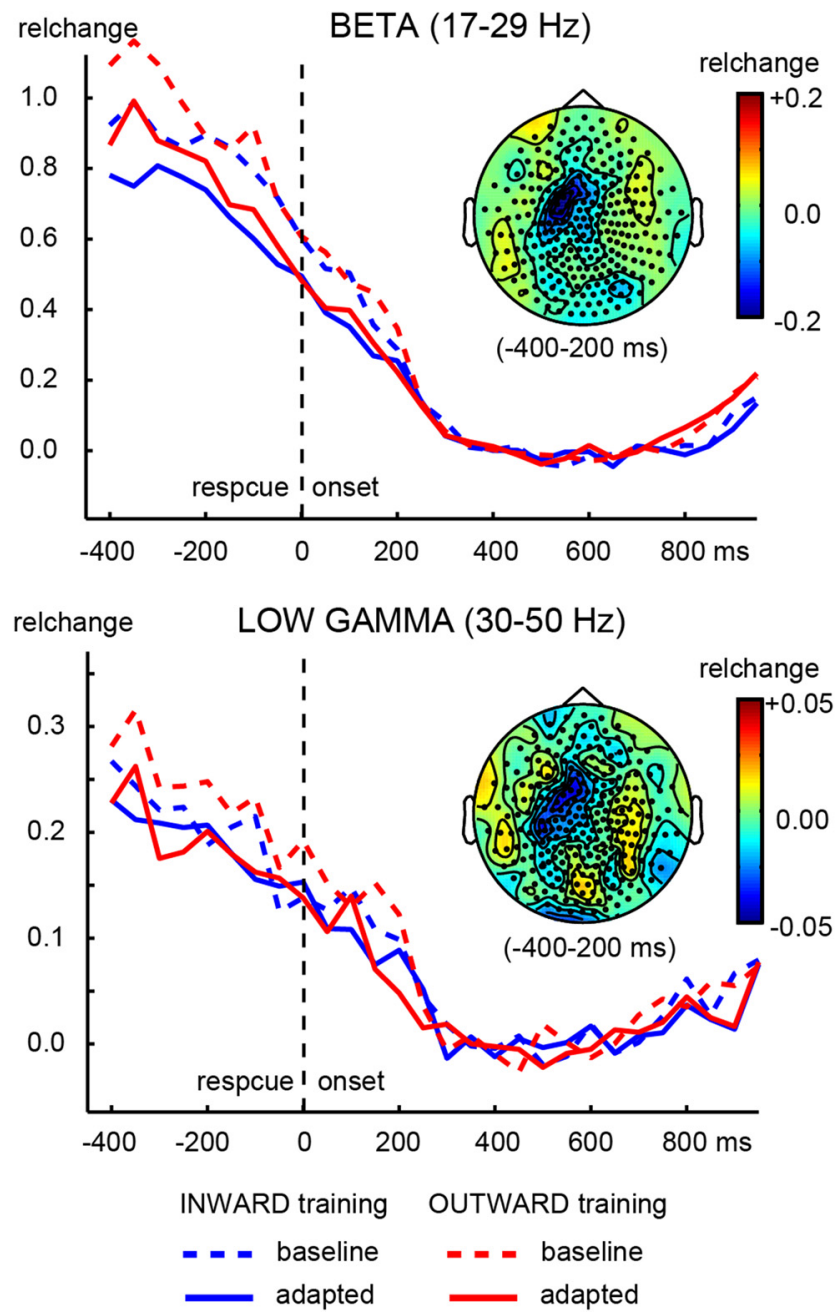

Figure 6. Effect of visuomotor adaptation on motor cortex oscillations. Grand-average traces of relative changes in beta (top) and low-gamma band (bottom) power of the LMtctx ROI, for data acquired before and after adaptation. The distribution of the adaptation-induced change in power corresponds with the LMtctx ROI.

fore adaptation is not lower or in between the power levels for the inward and outward training conditions, but higher. That is, for both adaptation directions, there should be a reduction in power compared with the baseline power before adaptation, whereas this reduction might be larger for the inward than for the outward training. As illustrated in Figure 6, adaptation produced a reduced beta power during the intertrial period compared with the baseline phase. A two-factor ANOVA with factors TRAINING (baseline, adapted) and CONDITION (inward, outward) confirmed a main effect of TRAINING $\left(F_{(1,17)}=9.4, p=0.007\right)$ (test window $\left.-400-200 \mathrm{~ms}\right)$. The effect of CONDITION and the interaction TRAINING by CONDITION were not significant (both $F_{(1,17)}<1$ ). As Figure 6 shows, the data for the low-gamma-band activity were weaker. Nevertheless, the effect of TRAINING approached significance $\left(F_{(1,17)}=4.3, p=0.054\right)$. As for the beta band, the effect of CONDITION and the interaction TRAINING by CONDITION were not significant (both $F_{(1,17)}<1$ ). The distribution of the adaptation-induced reductions in power coincides with the motor cortex ROI.

Together, these analyses of the training data provide critical support for our hypothesis by confirming the prediction, based on Paz et al. (2003), that visuomotor adaptation pro- duces a reduction of motor cortex beta- (and, less conclusively, low-gamma) power, indicating increased neural activity associated with the learned movement directions. The data fall short of reproducing the stronger power reduction for the inward than for the outward adaptation conditions, the finding in the main experiment that we seek to explain. This is readily accounted for by the low number of trials and the absence of the delay period that reduced the observation window. Moreover, the motor cortex neural changes observed by Paz et al. (2003) occurred only in advanced phases of learning.

\section{Discussion}

Visuomotor adaptation is a widely used experimental tool for the investigation of mechanisms underlying learning and behavioral and brain plasticity (Krakauer and Mazzoni, 2011). It has also been used in combination with cell recordings in motor structures to evaluate visual and motor aspects of motor cortical activity (Alexander and Crutcher, 1990; Shen and Alexander, 1997a,1997b). The present study used an experimentally challenging form of dual adaptation in a novel application of this technique: to distinguish visual and motor aspects of motor cortex activation for multiple reach targets manipulated by varying their spatial separation.

\section{Target separation and neural competition}

Extensive work by Ghez et al. (1997) has demonstrated that reaching movements to one of two targets are influenced by the spatial separation between the two target locations. This effect of target separation has been modeled in terms of competing groups of neurons, with the strength and balance of competitive and cooperative interactions depending on the separation (Erlhagen and Schöner, 2002; Cisek, 2006; Pastor-Bernier and Cisek, 2011). These models have been developed, not merely to explain behavior in laboratory tasks, but to explore to what extent choice behavior may have evolved from and still uses neural circuitry for simple sensorimotor interactive behavior (Cisek and Kalaska, 2010). Some of the available experimental evidence supporting that decisions are reached through a mechanism of biased competition derives from a manipulation of target separation. PastorBernier and Cisek (2011) found that neural activity, cell firing rates in the dorsal premotor cortex, was much weaker for action choices at wide than at small separation, indicating greater competition. In addition, the activities associated with competing choices were sensitive to the value of the choice, but only relative to the competing choice.

Extending this work, and the associated model of biased competition, from monkey neurophysiology to humans, noninvasive methods suffer from the limitation that competing movement representations cannot be probed separately. Therefore, inferences on the strength of competition are necessarily based on the net activity of the motor cortex (Praamstra et al., 2009; Tzagarakis et al., 2010; Grent-'t-Jong et al., 2014). This renders the observation of stronger movement preparatory activity at small compared with larger target separation more vulnerable to alternative interpretations, specifically to the view that larger target separation (i.e., greater response uncertainty) reduces the inclination to prepare for movement. Using dual adaptation in the present study, we attempted to unpick the notion of response uncertainty, separating it into the objective uncertainty of the physical movement environment and the subjective uncertainty imparted on the mind of the participant, mainly through the visual appearance of target separation. This approach yielded results that support the role of competitive interactions between alternative 
movement plans in the motor cortex and the expression of this in net motor cortical activity measured at the scalp. However, the support was not based on an independent evaluation of visual and motor aspects of target separation as designed, but on changes in power of motor cortical beta and low-gamma rhythms related to visuomotor adaptation that were sensitive to the angle between the learned movement directions.

\section{Visuomotor adaptation and the sensorimotor cortex}

Our study used dual adaptation requiring a rotation in one direction in one part of the workspace and a rotation in the opposite direction in another part. Not only is the motor cortex involved in visuomotor adaptation, it has also been shown capable of simultaneously representing such conflicting sensorimotor mappings (Zach et al., 2012; Ogawa and Imamizu, 2013). This capability and the feasibility of dual adaptation are likely to be linked to the local nature of neural changes associated with the learning of a new visuomotor mapping (Paz et al., 2003; Zach et al., 2012). Such changes consist of an increase in activity of only cells with directional properties corresponding to the learned movement direction. Of key relevance to the present study is that this neural correlate of learning a new mapping persisted across an experimental block with the default mapping (Paz et al., 2003). We propose that the persistent nature of the increased firing rates is the explanation of the baseline differences in power during the intertrial period.

The differences in baseline activity between conditions were such that conditions with inward adaptation showed lower beta and low-gamma power than conditions with outward adaptation, indicating more preparatory activity for the former compared with the latter. The inward adaptation conditions involved learned movement directions that reduced the target separation, whereas the outward adaptation conditions were associated with new learning of movements at a wider angle than visually displayed. Crucially, the angle separating the learned movement directions was smaller in the inward than in the outward adaptation conditions. This means that the increased baseline activity (lower beta and low gamma power) for the inward versus the outward adaptation conditions can now be understood as the result of persistently increased activity (firing rate) of neural populations that encode movement directions at a closer angle in the inward than in the outward adaptation conditions. That is, due to the distance dependence of lateral interactions (i.e., altered balance between short-range mutual excitation and long-range lateral inhibition; Cisek, 2006; Pastor-Bernier and Cisek, 2011), the difference in angle of learned movement directions translates into a difference in sustained activity of the motor cortex.

The effect of visuomotor adaptation on sustained oscillatory activity of the motor cortex prohibits the selective evaluation of visual and movement target separation effects as originally designed. Fortuitously, the dependence of the sustained effect on the separation between (learned) movement targets turns this effect into a finding that speaks to the original question. The influence of movement target separation overrides the influence of visual target separation because the visual targets are at $30^{\circ}$ and $60^{\circ}$ separation in the outward adaptation conditions and at a net greater separation of $60^{\circ}$ and $90^{\circ}$ in the inward adaptation conditions. Moreover, as a sustained effect, instead of an effect occurring after display of movement targets in a delay period only, it rules out that higher movement preparatory activity for targets at small compared with large separation is due to subjects being more inclined to prepare for movement.

\section{Conclusion}

Eisenberg et al. (2010) compellingly demonstrated that clustering of motor cortex cells with similar directional preference is detectable with MRI at the voxel level. The present results show that this architecture can be exploited to study the interactions between cell groups encoding alternative actions using noninvasive MEG measurements. The results support that spatial variables of competing actions have a direct influence on sensorimotor cortex activity, providing important support for biased competition models of action selection (Cisek and Kalaska, 2010; PastorBernier and Cisek, 2011). Furthermore, using dual visuomotor adaptation, the investigation produced evidence in support of the local nature of neural changes related to the learning and retention of new visuomotor mappings (Paz et al., 2003). The expression of such changes in sustained effects on oscillatory power of motor cortex rhythms makes mechanisms of visuomotor transformations better accessible for study in human subjects.

\section{References}

Alexander GE, Crutcher MD (1990) Neural representations of the target (goal) of visually guided arm movements in three motor areas of the monkey. J Neurophysiol 64:164-178. Medline

Bastiaansen MC, Knösche TR (2000) Tangential derivative mapping of axial MEG applied to event-related desynchronization research. Clin Neurophysiol 111:1300-1305. CrossRef Medline

Bastian A, Schöner G, Riehle A (2003) Preshaping and continuous evolution of motor cortical representations during movement preparation. Eur J Neurosci 18:2047-2058. CrossRef Medline

Bock O, Eversheim U (2000) The mechanisms of movement preparation: a precuing study. Behav Brain Res 108:85-90. CrossRef Medline

Cisek P (2006) Integrated neural processes for defining potential actions and deciding between them: a computational model. J Neurosci 26:97619770. CrossRef Medline

Cisek P, Kalaska JF (2005) Neural correlates of reaching decisions in dorsal premotor cortex: specification of multiple direction choices and final selection of action. Neuron 45:801-814. CrossRef Medline

Cisek P, Kalaska JF (2010) Neural mechanisms for interacting with a world full of action choices. Annu Rev Neurosci 33:269-298. CrossRef Medline

Doyle LM, Yarrow K, Brown P (2005) Lateralization of event-related beta desynchronization in the EEG during pre-cued reaction time tasks. Clin Neurophysiol 116:1879-1888. CrossRef Medline

Eisenberg M, Shmuelof L, Vaadia E, Zohary E (2010) Functional organization of human motor cortex: directional selectivity for movement. J Neurosci 30:8897-8905. CrossRef Medline

Erlhagen W, Schöner G (2002) Dynamic field theory of movement preparation. Psychol Rev 109:545-572. CrossRef Medline

Gallivan JP, Barton KS, Chapman CS, Wolpert DM, Randall Flanagan J (2015) Action plan co-optimization reveals the parallel encoding of competing reach movements. Nat Commun 6:7428. CrossRef Medline

Ghez C, Favilla M, Ghilardi MF, Gordon J, Bermejo R, Pullman S (1997) Discrete and continuous planning of hand movements and isometric force trajectories. Exp Brain Res 115:217-233. CrossRef Medline

Grent-'t-Jong T, Oostenveld R, Jensen O, Medendorp WP, Praamstra P (2013) Oscillatory dynamics of response competition in human sensorimotor cortex. Neuroimage 83:27-34. CrossRef Medline

Grent-'t-Jong T, Oostenveld R, Jensen O, Medendorp WP, Praamstra P (2014) Competitive interactions in sensorimotor cortex: oscillations express separation between alternative movement targets. J Neurophysiol 112:224-232. CrossRef Medline

Hämäläinen M, Hari R, Ilmoniemi RJ, Knuutila J, Lounasmaa OV (1993) Magnetoencephalography: theory, instrumentation, and applications to noninvasive studies of the working human brain. Reviews of Modern Physics 65:413-497. CrossRef

Hinder MR, Riek S, Tresilian JR, de Rugy A, Carson RG (2010) Real-time error detection but not error correction drives automatic visuomotor adaptation. Exp Brain Res 201:191-207. CrossRef Medline

Klaes C, Westendorff S, Chakrabarti S, Gail A (2011) Choosing goals, not rules: deciding among rule-based action plans. Neuron 70:536-548. CrossRef Medline

Krakauer JW, Mazzoni P (2011) Human sensorimotor learning: adapta- 
tion, skill, andbeyond. Curr Opin Neurobiol 21:636-644. CrossRef Medline

Maris E, Oostenveld R (2007) Nonparametric statistical testing of EEG- and MEG-data. J Neurosci Methods 164:177-190. CrossRef Medline

Mitra PP, Pesaran B (1999) Analysis of dynamic brain imaging data. Biophys J 76:691-708. CrossRef Medline

Ogawa K, Imamizu H (2013) Human sensorimotor cortex represents conflicting visuomotor mappings. J Neurosci 33:6412-6422. CrossRef Medline

Oostenveld R, Fries P, Maris E, Schoffelen JM (2011) FieldTrip: Open source software for advanced analysis of MEG, EEG, and invasive electrophysiological data. Comput Intell Neurosci 2011:156869. Medline

Pastor-Bernier A, Cisek P (2011) Neural correlates of biased competition in premotor cortex. J Neurosci 31:7083-7088. CrossRef Medline

Pastötter B, Berchtold F, Bäuml KH (2012) Oscillatory correlates of controlled speed-accuracy tradeoff in a response-conflict task. Hum Brain Mapp 33:1834-1849. CrossRef Medline

Paz R, Boraud T, Natan C, Bergman H, Vaadia E (2003) Preparatory activity in motorcortex reflects learning of local visuomotor skills. Nat Neurosci 6:882-890. CrossRef Medline

Pellizzer G, Hedges JH (2003) Motor planning: effect of directional uncertainty with discrete spatial cues. Exp Brain Res 150:276-289. Medline

Praamstra P, Kourtis D, Nazarpour K (2009) Simultaneous preparation of multiple potential movements: opposing effects of spatial proximity mediated by premotor and parietal cortex. J Neurophysiol 102:2084-2095. CrossRef Medline
Rawle CJ, Miall RC, Praamstra P (2012) Frontoparietal theta activity supports behavioral decisions in movement-target selection. Front Hum Neurosci 6:138. Medline

Shen L, Alexander GE (1997a) Neural correlates of a spatial sensory-tomotor transformation in primary motor cortex. J Neurophysiol 77:11711194. Medline

Shen L, Alexander GE (1997b) Preferential representation of instructed target location versus limb trajectory in dorsal premotor area. J Neurophysiol 77:1195-1212. Medline

Stewart BM, Gallivan JP, Baugh LA, Flanagan JR (2014) Motor, not visual, encoding of potential reach targets. Curr Biol 24:R953-R954. CrossRef

Stolk A, Todorovic A, Schoffelen JM, Oostenveld R (2013) Online and offline tools for head movement compensation in MEG. Neuroimage 68: 39-48. CrossRef Medline

Tzagarakis C, Ince NF, Leuthold AC, Pellizzer G (2010) Beta-band activity during motor planning reflects response uncertainty. J Neurosci 30 : 11270-11277. CrossRef Medline

van Wijk BC, Daffertshofer A, Roach N, Praamstra P (2009) A role of beta oscillatory synchrony in biasing response competition? Cereb Cortex 19: 1294-1302. CrossRef Medline

Woolley DG, de Rugy A, Carson RG, Riek S (2011) Visual target separation determines the extent of generalisation between opposing visuomotor rotations. Exp Brain Res 212:213-224. CrossRef Medline

Zach N, Inbar D, Grinvald Y, Vaadia E (2012) Single neurons in M1 and premotor cortex directly reflect behavioral interference. PLoS One 7:e32986. CrossRef Medline 